\title{
THE IMPACT OF MASS FLOW AND MASKING ON THE PRESSURE DROP OF AIR FILTER IN HEAVY-DUTY DIESEL ENGINE
}

\author{
Sepideh HOSEEINZADEH ${ }^{1}$, Mofid GORJI-BANDPY ${ }^{2}$
}

\begin{abstract}
This paper presents a computational fluid dynamics (CFD) calculation approach to predict and evaluate the impact of the mass-flow inlet on the pressure drop of turbocharger's air filtfer in heavy-duty diesel engine. The numerical computations were carried out using a commercial CFD program whereas the inlet area of the air filter consisted of several holes connected to a channel. After entering through the channel, the air passes among the holes and enters the air filter. The effect of masking holes and hydraulic diameter is studied and investigated on pressure drop. The results indicate that pressure drop increase with decreasing of hydraulic diameter and masking of the holes has considerable affect on the pressure drop.
\end{abstract}

Keywords: Air filter, Mass flow, Pressure drop, Heavy-duty diesel engine

\section{Introduction}

Air cleaning devices for air-born particles are widely used in various industrial applications such as nuclear, automotive industry, food processing, and etc. Numerous models have been proposed to calculate the efficiency and the pressure drop of filters under steady flow conditions. The majorities of these models are only applicable to flat filters and not pleated filters. Moreover, there are few studies on the variation of these values in dynamic filtration. Kim et al. [1] studied temperature effects on the pressure drop at a ceramic filter. Pressure drop evolution of fibrous filter under continuous aerosol loading has been studied by Bergman et al.[2], Vendel et al.[3], Letourneau et al.[4], Novick et al.[5] and Thomas et al.[6] focusing on high-efficiency particulate air (HEPA) filters. Many authors have considered various aspects of this problem and obtained similar solutions [7-10]. Also Awniet al. [11] predicted efficiency, thickness, porosity and pressure drop at a new model of filter cake. Also several works are fulfilled in CFD field that shows the relation between the velocity and pressure drop. For example Gimbun et al. [12] was studied the influence of the temperature and inlet velocity on the cyclone pressure drop. One application of the air filter is in the heavy-duty diesel engine. Engineers are generally interested in two parameters in order to carry out an assessment of the design and performance of air filters.

1: Hosseinzadeh Sepideh- Address: No96, Allay 2,St 6, Daryakenar, Babolsar, Mazandaran, IranEmail:sepideh_h87@yahoo.com

2: Gorji-BandpyMofid -Address: Department of Mechanical Engineering, Babol University of Technology, P.O. Box 484, Babol, Iran- Email: gorji@nit.ac.ir 
These parameters are the temperature and pressure drop through the air filter. In this work, the effect of mass flow rate inlet on pressure drop was studied. Accurate prediction of air filter pressure drop is very important, because it relates directly to operating costs.

In this work, the air filter in heavy-duty diesel engine that is shown in Figure. 1 is studied This air filter is consisted of nine solid circular plate whereas these plates act as cleaners and also chokers. The inlet area of the air filter consists of many holes. In this air filter, air after crossing a channel enters into the aforementioned holes that creates turbulent flow in the vacant space and then crosses among the plates.

Inlet area of this air filter contains many holes that is connected to a channel. The air, with various mass flow rates, enters the channel and passes through the holes and then enters the filter. In the first section of our study, the effect of the mass flow inlet and percentage of the masking holes on pressure drop are studied. In this section all of the holes are ellipse shaped and in per stage several rows of the holes were closed from the top to bottom.

In the second section of our study, the circles shaped are studied; and it is assumed that all of the holes are opened. The study is performed for three different hydraulic diameters and the results are compared.

In the third step of our work, the pressures drop obtained under different mass flow inlets for the inlet shape studied with different hydraulic diameters are compared.

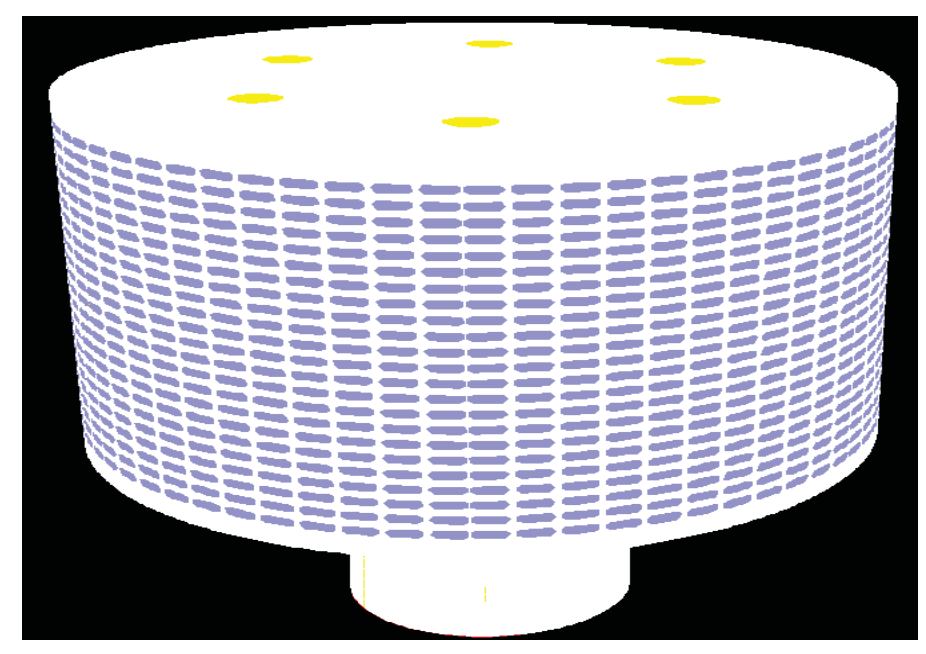

Figure1: The complete shape of the Air filter with ellipse shapes as inlet area 


\section{NUMERICAL MODEL SETUP}

\section{1 STUDY MODEL}

In this work, the three-dimension geometry of the air filter was generated and the meshing of the volumes was done using Gambit. Only a sector of $60^{\circ}$ of this geometry filter was modeled and meshed, due to huge volume of the air filter. Tetrahedron meshes with $1.2,1.5,1.8$ and 2.1 million elements were generated within the geometry by the CFD software. Preliminary tests showed that the meshing with1.5 million elements was the one that gave the best compromise between quality of mesh element (in terms of aspect ratio and skewness), CPU time, and numerical dispersion introduced by the spatial discretization method. Eventually, obtained pressure drops were extended to the whole of the air filter and are depicted in charts in the next section. Inlet area in air filter was connected to a channel with a height and radius of 360 and800 mm, respectively. Meshing for the air filter connected to a channel is shown in Figure. 2.

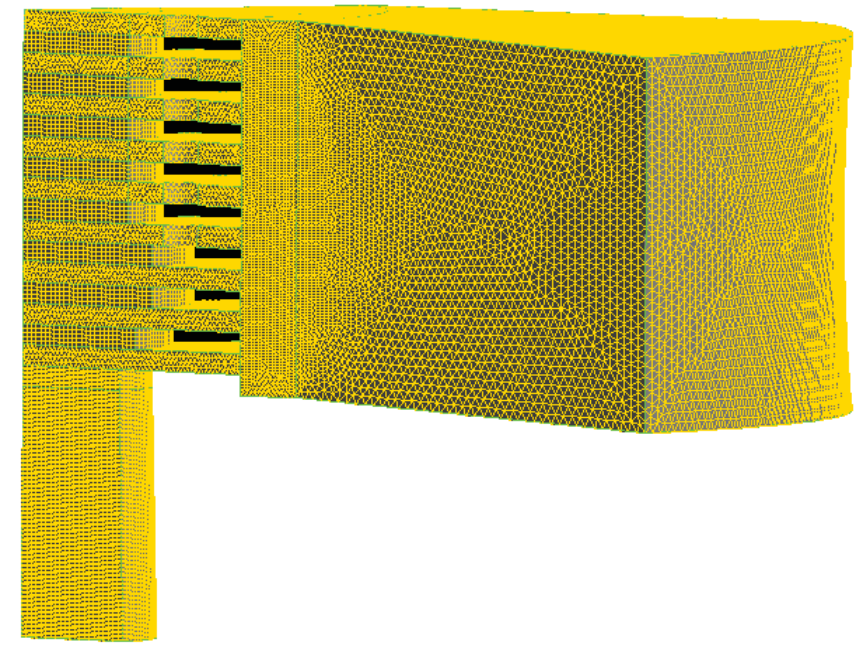

Figure 2: Computational mesh for the air filter with a channel

\section{2 BOUNDARY CONDITIONS}

The boundary conditions are illustrated in Figure.3, and described below with the following initial conditions;

- The mass flow inlet at the entrance of the channel is defined as turbulent in the normal direction with different mass flow rates.

- The outflow of the air filter was defined as outlet pressure and was assumed to be zero.

- The side of the air filter and channel were defined as periodic.

- Other faces of air filter were defined as wall.

- The fluid was assumed to be incompressible and ideal gas.

- The temperatures at all of the faces were assumed to be constant. 


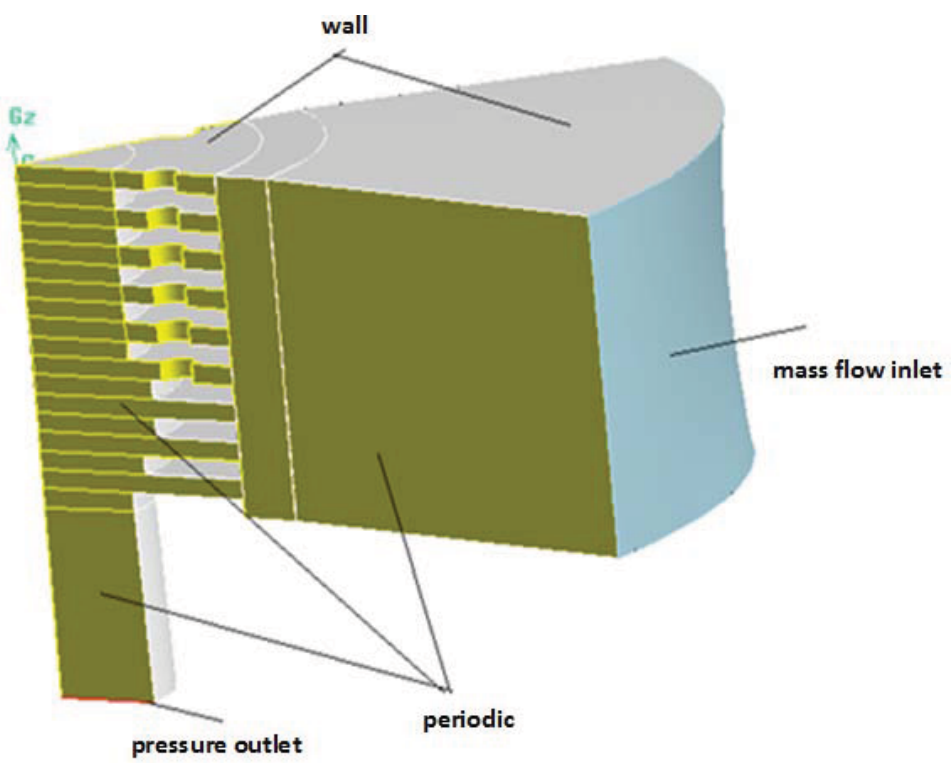

Figure3: Air filter`s boundary conditions for CFD calculations

\section{3 CONSERVATION EQUATIONS}

The complicated swirling turbulent flow in the air filter demands on numerical techniques and turbulence models employed in the CFD code when modeling the air filter pressure drop.

In this analysis commercial CFD software package, Fluent v.6.3, was used to predict the velocity profile and pressure drop inside the air filter. Under turbulent flow condition, a modification of the realizable $\mathrm{K}-\varepsilon$ model was employed with standard wall function. In all of our simulations the fluid was considered to be Newtonian, incompressible, ideal gas and the properties of the fluid were assumed to be constant . The mass conservation equation, or continuity equation, can be written as flow:

$$
\partial \rho / \partial \mathrm{t}+\nabla \cdot(\rho \vec{u})=S_{m}
$$

This is the general form of the mass conservation equation. Here the term $S_{m}$ , designates the contribution of mass from another dispersed phase and is equal to zero, $\rho$ is the fluid density and uisthe velocity vector. For an incompressible fluid the continuity equation simplifies to $\operatorname{div} \overrightarrow{\mathrm{u}}=0$. The conservation the momentum in inertial (nonaccelerating) reference frame can be written as follow:

$$
\partial / \partial \mathrm{t}(\rho \overrightarrow{\mathrm{u}})+\nabla \cdot(\rho \overrightarrow{\mathrm{uu}})=-\nabla \mathrm{p}+\nabla \cdot(\overline{\bar{\tau}})+\rho \overrightarrow{\mathrm{g}}+\overrightarrow{\mathrm{F}}
$$

where $\mathrm{p}$ is the static pressure, $\overline{\bar{\tau}}$ is the stress tensor (describes below), and $\rho \vec{g}$ and $\vec{F}$ are the gravitational body force and the external body forces, respectively. For the Newtonian fluid, the stress tensor is given by

$$
\overline{\bar{\tau}}=\mu\left[\left(\nabla \overrightarrow{\mathrm{u}}+\nabla \overrightarrow{\mathrm{u}}^{\mathrm{T}}\right)-2 / 3 \nabla \cdot \overrightarrow{\mathrm{u}} \mathrm{I}\right]
$$

where $\mu$ is the dynamics viscosity, I is the unit tensor, and the second term in the right side inside the square brackets is the effect of volume dilation and for incompressible fluid it is equal to zero. Turbulence was modeled using the realizable $k-\varepsilon$ model, since it 
has been shown to give accurate results for flows involving rotation, boundary layer under strong adverse pressure gradients, separation and recirculation [13]. In this model the transport equations for $\mathrm{k}$ (turbulence kinetic energy) and the $\varepsilon$ (turbulence kinetic energy dissipation rate) are as follows;

$$
\begin{gathered}
\frac{\partial}{\partial t}(\rho \mathrm{k})+\frac{\partial}{\partial \mathrm{x}_{\mathrm{j}}}\left(\rho k u_{\mathrm{j}}\right)=\frac{\partial}{\partial \mathrm{x}_{\mathrm{j}}}\left[\left(\mu+\frac{\mu_{\mathrm{t}}}{\sigma_{k}}\right) \frac{\partial k}{\partial x_{j}}\right]+G_{k}+G_{b}-\rho \varepsilon \\
\frac{\partial}{\partial \mathrm{t}}(\rho \varepsilon)+\frac{\partial}{\partial \mathrm{x}_{\mathrm{j}}}\left(\rho \varepsilon \mathrm{u}_{\mathrm{j}}\right)=\frac{\partial}{\partial \mathrm{x}_{\mathrm{j}}}\left[\left(\mu+\frac{\mu_{\mathrm{T}}}{\sigma_{\varepsilon}}\right)\right]+\rho \mathrm{C}_{1} \mathrm{~S}_{\varepsilon}-\rho \mathrm{C}_{2} \frac{\varepsilon^{2}}{\mathrm{k}+\sqrt{\vartheta \varepsilon}}+\mathrm{C}_{1 \varepsilon} \frac{\varepsilon}{\mathrm{k}} \mathrm{C}_{2 \varepsilon} \mathrm{G}_{\mathrm{b}}+\mathrm{S}_{\varepsilon}
\end{gathered}
$$

and the adjustable parameters are:

$$
\begin{aligned}
& \mu_{T}=\rho C_{\mu} k^{2} / \varepsilon \\
& C_{1}=\max [0.43, \eta /(\eta+5)] \\
& \eta=S \mathrm{~K} / \varepsilon \\
& S=\sqrt{2 S_{i j} S_{i j}}
\end{aligned}
$$

In this model $G_{k}$ represents the generation of turbulence kinetic energy due to the mean velocity gradients, $G_{b}$ is the generation of turbulence kinetic energy due to buoyancy, $\sigma_{k}$ and $\sigma_{\varepsilon}$ are the turbulent Prandtl numbers for $\mathrm{k}$ and $\varepsilon ; S_{i j}$ is the mean strain rate tensor and $\Omega_{i j}$ is the mean rate of in a rotating reference frame with angular velocity $\overline{\omega_{k}}$. From these equations one can see that $C_{\mu}$ is not constant, it varies with mean strain and rotation rates. The model constants, $C_{1 \varepsilon}, C_{2}$, and $\sigma_{k}$ and $\sigma_{\varepsilon}$ are assigned the following values; $C_{1 \varepsilon}=1.44, C_{2}=1.9, \sigma_{k}=1.0, \sigma_{\varepsilon}=1.2$; these values have been established to ensure that the model performs well for certain canonical flows[14].

\section{RESULTS AND DISCUSSION}

\section{Pressure drop prediction under different mass flow inlet and masking holes}

Pressure drop was measured at four mass flow rates and five percentage of masking by the CFD and the results are shown in Figure.7. Mass flow rate ranges from $1 \mathrm{~kg} / \mathrm{s}$ to 4 $\mathrm{kg} / \mathrm{s}$. At mass flow rate of $1 \mathrm{~kg} / \mathrm{s}$ the pressure drop rises from $0.376 \mathrm{KPa}$ at $0 \%$ masking to $0.939 \mathrm{KPa}$ at74\%masking. At mass flow rate of $2 \mathrm{~kg} / \mathrm{s}$ the pressure drop varies from $1.486 \mathrm{KP}$ to $3.622 \mathrm{Kpa}$ at $0 \%$ and $74 \%$ respectively. Also it can be seen that the same value soars from $3.316 \mathrm{KPa}$ to $8.108 \mathrm{KPa}$ and $5.789 \mathrm{KPa}$ to $13.909 \mathrm{Kpa}$ at mass flow rate of $3 \mathrm{~kg} / \mathrm{s}$ and $4 \mathrm{~kg} / \mathrm{s}$ for $0 \%$ and $74 \%$ masking respectively.

As can be seen, the pressure drop increases as mass flow rate rises. It can also be observed that for masking of $50 \%$ and higher, pressure drops increase more dramatically than the same value for masking of less than $50 \%$ which is not considerable.

The three -dimension contour of static pressure and velocity vectors in two cases were shown in Figures.5 and 6, respectively. As is seen in Figure.6 where no masking is implemented created vortex at mass flow rate of $4 \mathrm{~kg} / \mathrm{s}$ of more than mass flow rate of $1 \mathrm{~kg} / \mathrm{s}$. Also when $74 \%$ of the holes are masked the same condition can be seen. But when $74 \%$ of the holes are closed the created vortex at both mentioned mass flow rates is far more than the $0 \%$ masking. 


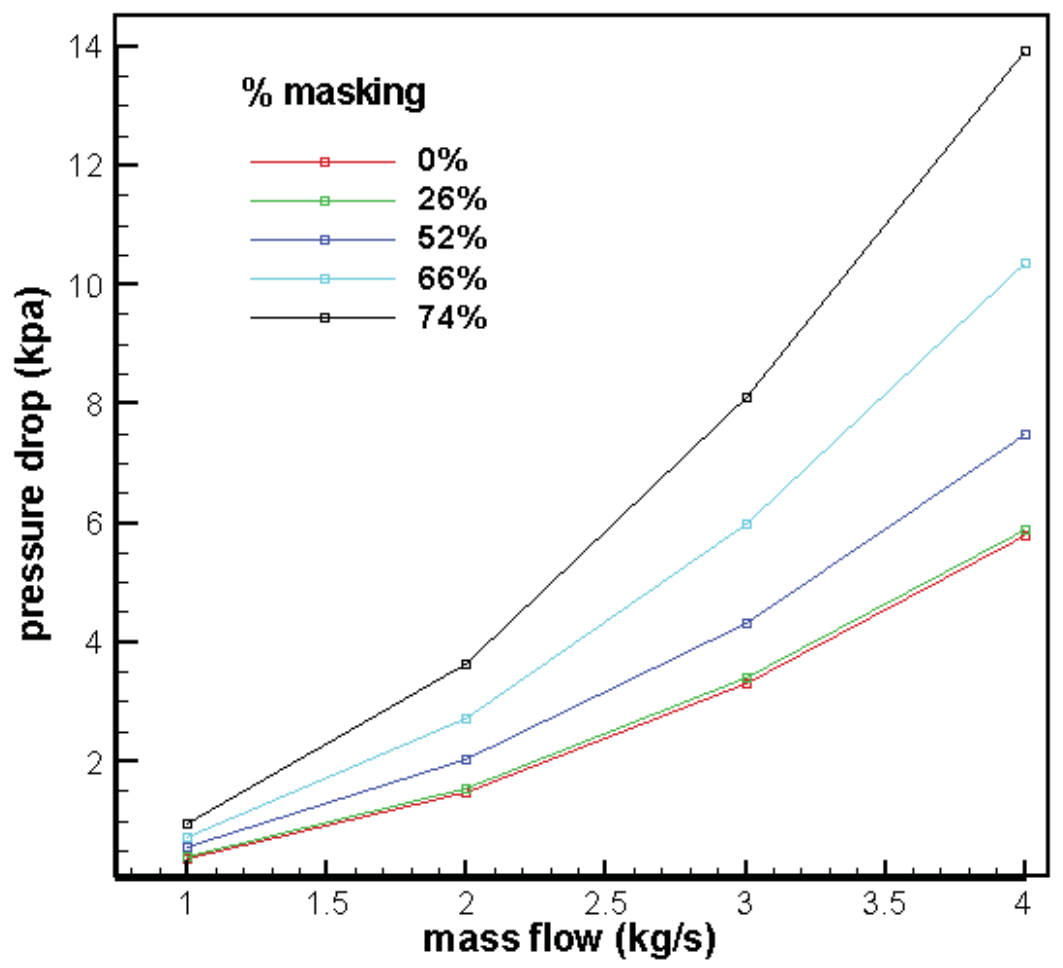

Figure 4: Altering pressure drop with variety of mass flow at different masking holes at air filter

\section{Pressure drop prediction under different mass flow inlet and different diameter}

This section was predicted the air filter's pressure drop when the shape of the holes alter to circle holes. All of the holes were opened and the air crosses the channel with mass flow inlet ranging from 1 to $4 \mathrm{~kg} / \mathrm{s}$. In this section pressure drop in three different diameters were compared and was seen that the decreasing of diameter leads to raising the pressure drop. This rising was seen with high rate when the diameters change from 12.4 to $6.2 \mathrm{~mm}$. In Figure.7 the pressure drop at three different diameters were shown. 
(a)
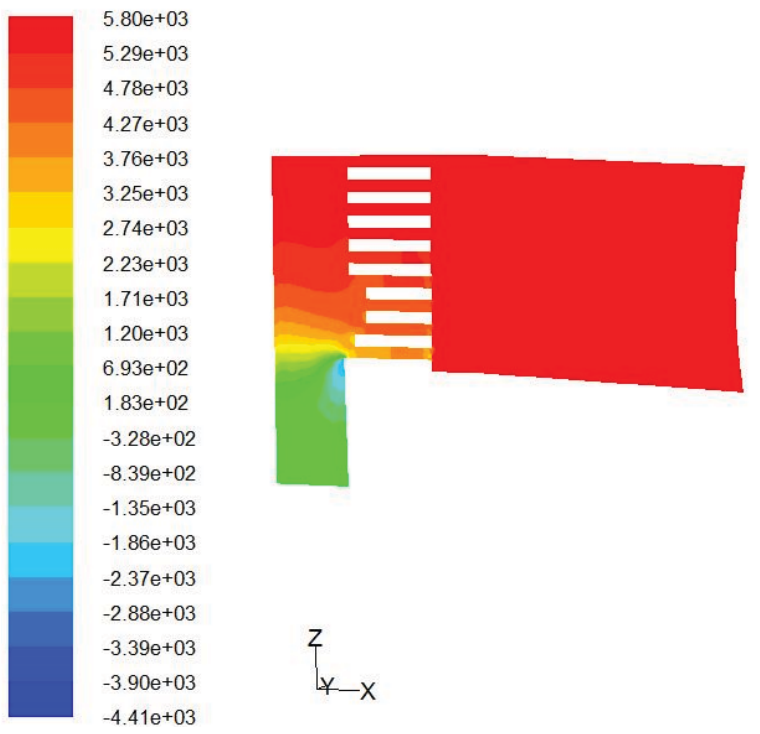

(b)

$$
\left\{_{y-x}\right.
$$

(d)

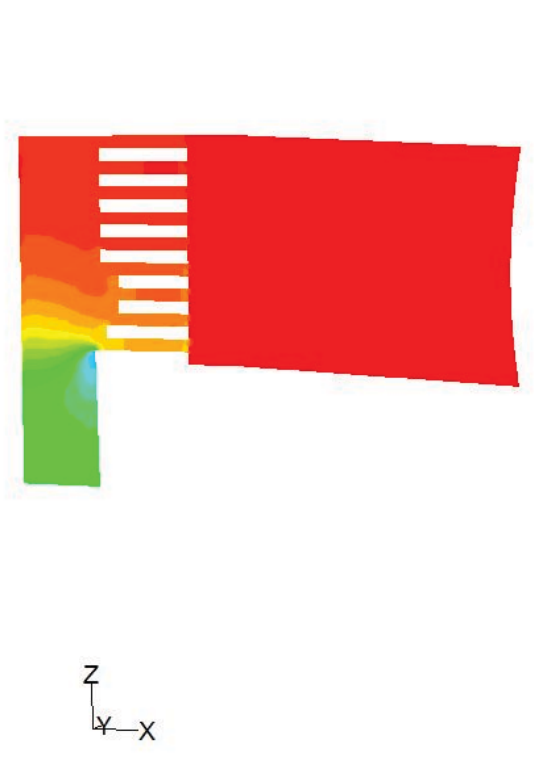

(c)
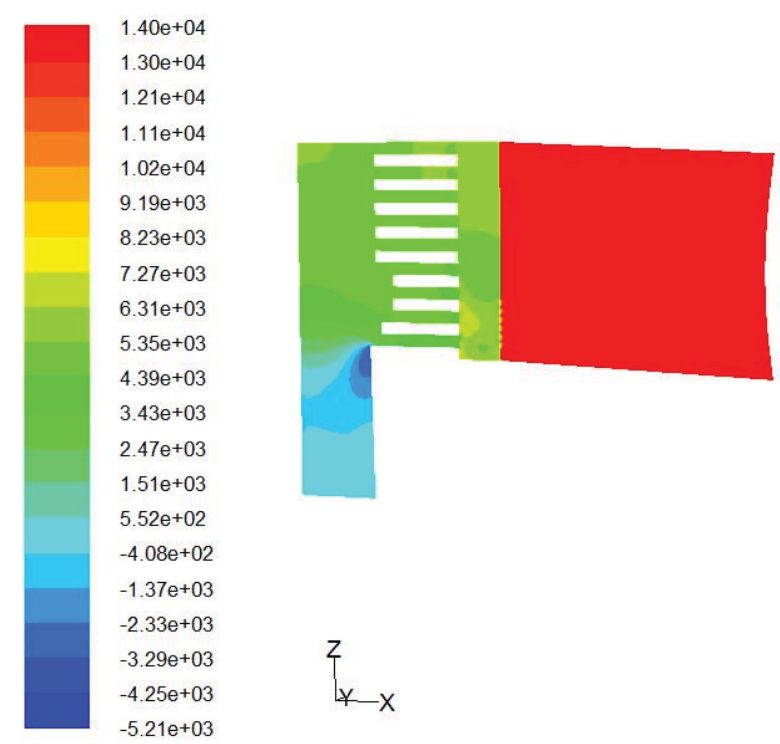

$$
\left\{_{y-x}\right.
$$

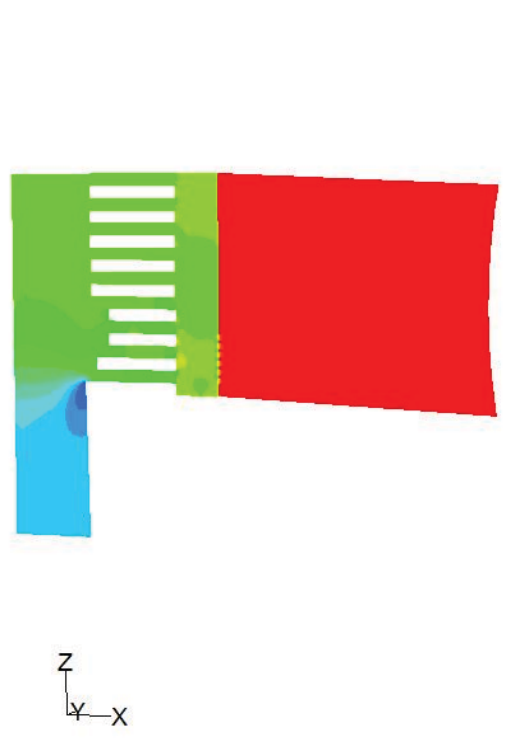

$6.39 \mathrm{e}+01$

6.59e- 01

$-6.26 \mathrm{e}+01$

$-1.26 \mathrm{e}+02$

$-1.89 \mathrm{e}+02$

$-2.52 \mathrm{e}+02$

$-3.15 \mathrm{e}+02$

Figure5: Contour of static pressure (Pascal)(a)for mass flow of $4 \mathrm{~kg} / \mathrm{s}$ at $0 \%$ masking of holes (b)for mass flow of $1 \mathrm{~kg} / \mathrm{s}$ at $0 \%$ masking of holes (c)for mass flow of $4 \mathrm{~kg} / \mathrm{s}$ at $74 \%$ masking of holes (d)for mass flow of $1 \mathrm{~kg} / \mathrm{s}$ at $74 \%$ masking of holes 
(a)

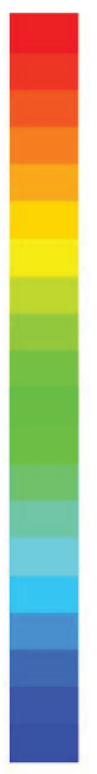

$9.89 \mathrm{e}+01$

$9.40 \mathrm{e}+01$

$8.90 \mathrm{e}+01$

$8.41 \mathrm{e}+01$

$7.92 \mathrm{e}+01$

$7.42 \mathrm{e}+01$

$6.93 \mathrm{e}+01$

$6.43 e+01$

$5.94 \mathrm{e}+01$

$5.44 \mathrm{e}+01$

$4.95 \mathrm{e}+01$

$4.45 \mathrm{e}+01$

$3.96 e+01$

$3.46 \mathrm{e}+01$

$2.97 \mathrm{e}+01$

$2.47 e+01$

$1.98 \mathrm{e}+01$

$1.49 \mathrm{e}+01$

$9.91 e+00$

$4.97 \mathrm{e}+00$

$1.94 \mathrm{e}-02$

(b)
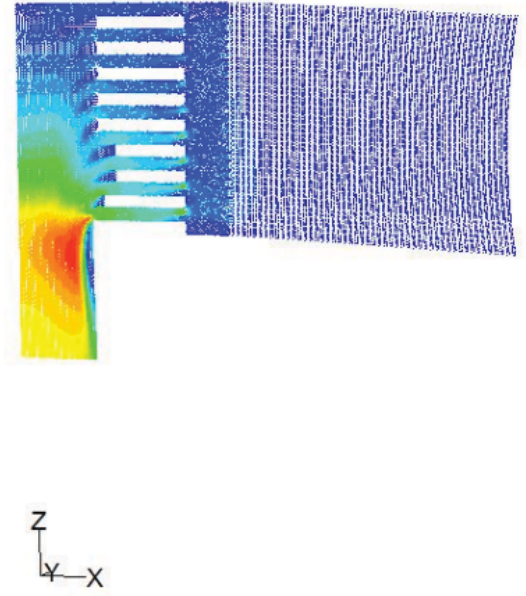

(d)

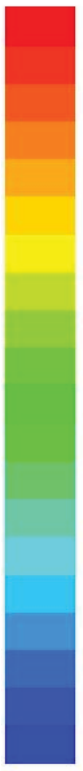

$2.47 \mathrm{e}+01$

$2.35 \mathrm{e}+01$

$2.22 \mathrm{e}+01$

2. $10 \mathrm{e}+01$

$1.98 \mathrm{e}+01$

$1.85 \mathrm{e}+01$

$1.73 e+01$

$1.61 \mathrm{e}+01$

$1.48 \mathrm{e}+01$

$1.36 \mathrm{e}+01$

$1.24 \mathrm{e}+01$

$1.11 \mathrm{e}+01$

$9.89 e+00$

$8.65 \mathrm{e}+00$

$7.42 \mathrm{e}+00$

$6.18 \mathrm{e}+00$

$4.95 \mathrm{e}+00$

$3.71 \mathrm{e}+00$

$2.48 \mathrm{e}+00$

$1.24 \mathrm{e}+00$

9.82e-03

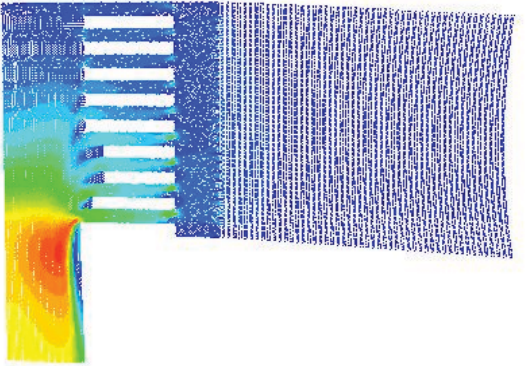

Figure 6: Velocity vector for air filter (a)for mass flow of $4 \mathrm{~kg} / \mathrm{s}$ at $0 \%$ masking of holes (b)for mass flow of $1 \mathrm{~kg} / \mathrm{s}$ at $0 \%$ masking of holes (c)for mass flow of $4 \mathrm{~kg} / \mathrm{s}$ at $74 \%$ masking of holes (d)for mass flow of $1 \mathrm{~kg} / \mathrm{s}$ at $74 \%$ masking of holes

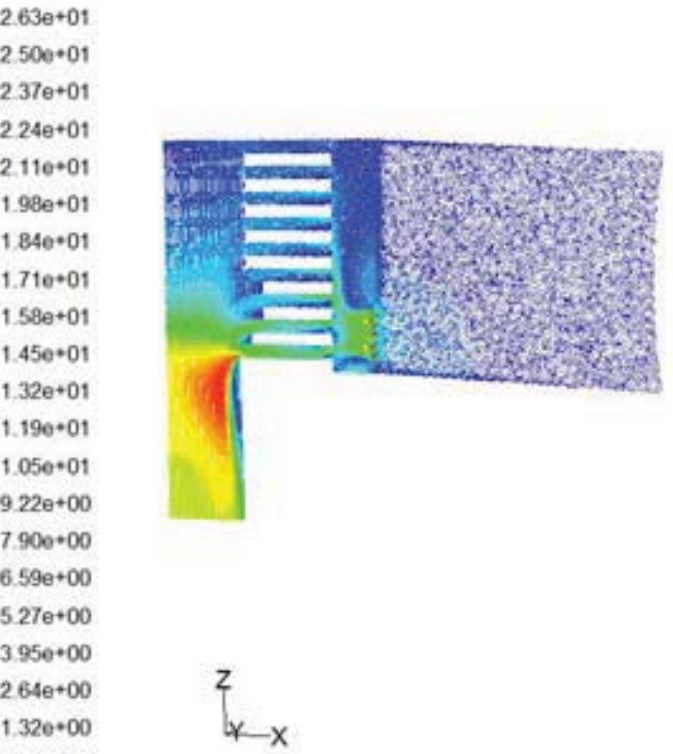

$1.320 * 00$

$1.510-03$

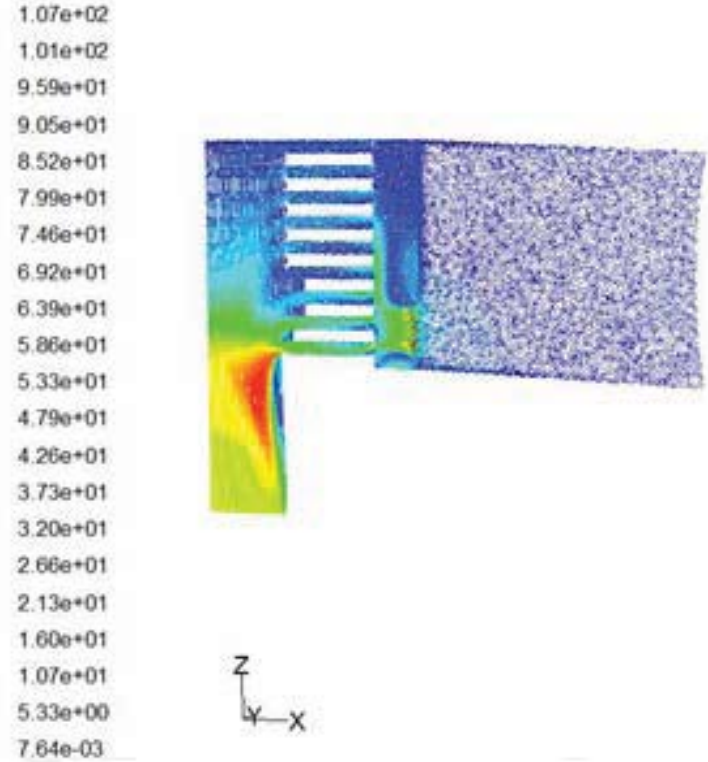

(c)

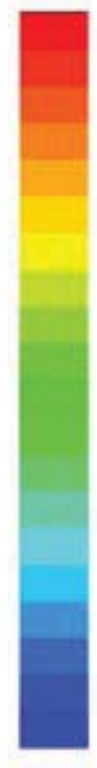


3. Pressure drop prediction under different mass flow inlet for two inlet shapes with different hydraulic diameters

In the third section the effect of the mass flow inlet on the pressure drop at the air filter between two different inlet shapes (ellipse and circle) was investigated. In both cases, all of the holes are open. In this state the hydraulic diameter for ellipse is $10.6 \mathrm{~mm}$ and for circle is $24.8 \mathrm{~mm}$, but the numbers of the ellipse approximately are more than doubled of the number of circles. In Figure. 8 it is resulted that the pressure drop resulting the variety of mass flow inlet in both cases are equal. It was shown that if the total hydraulic diameters at air filter in both cases be considered, the pressure drop is independent of the shape of the inlet holes.

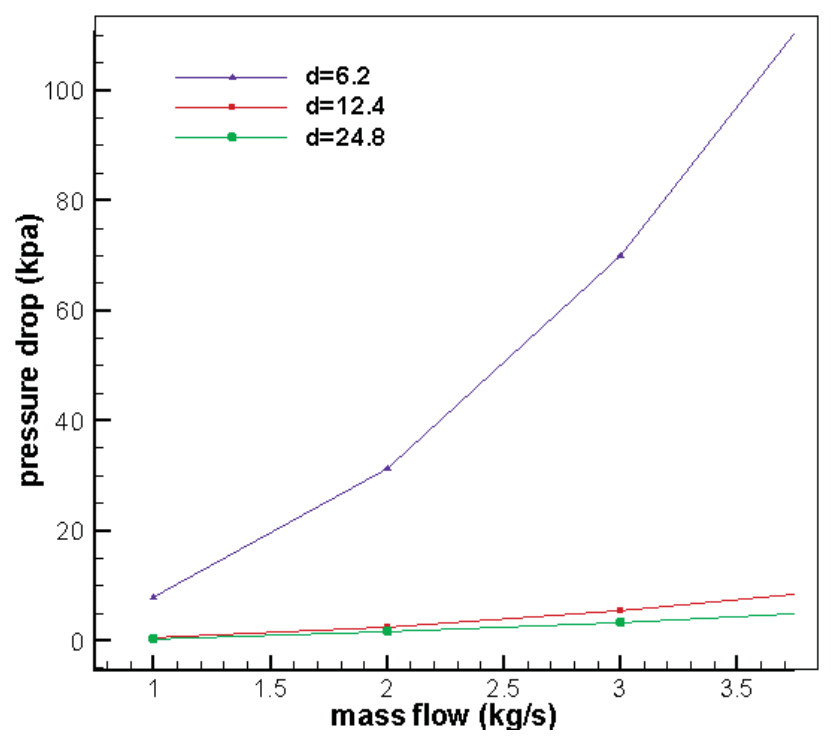

Figure7: Altering pressure drop for different two kind of diameters

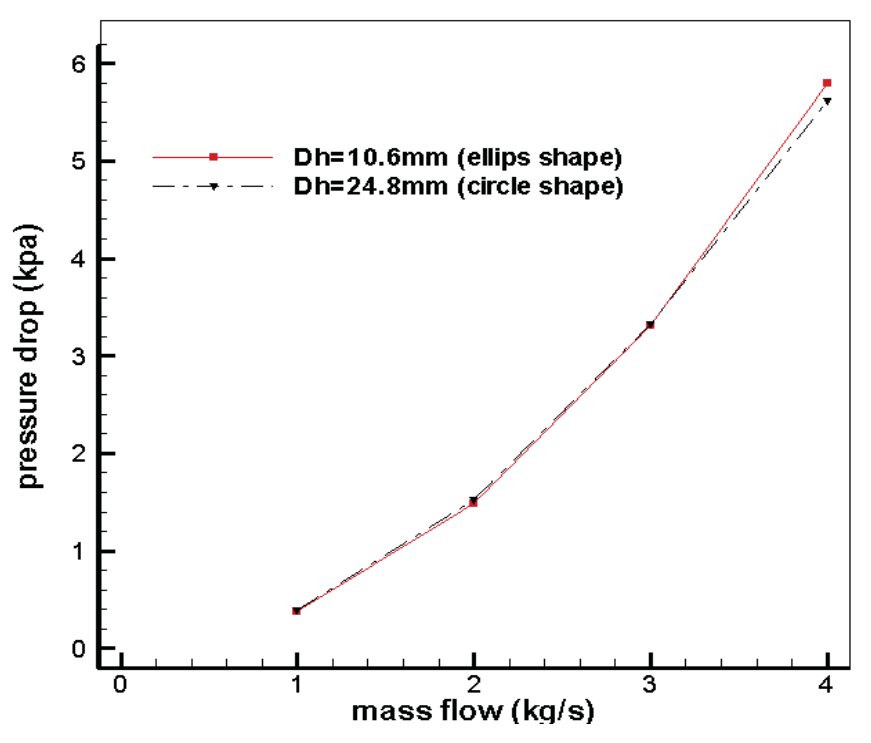

Figure8: Altering pressure drop with shape as an inlet area

\section{Conclusions}

In this paper a computational fluid dynamics (CFD) calculation approach to predict and evaluate the impact of the mass-flow inlet on the pressure drop of turbocharger's air filter in heavy-duty diesel engine was investigated. The CFD code with the realizable k- $\varepsilon$ turbulence model, predict the pressure drop in air filter with variety shapes with different hydraulic diameter as an inlet area. It can clearly be seen that pressure drops depends on mass flow and hydraulic diameter. And it can also be concluded that the pressure drop independent of the shape of the holes in air filter. 


\section{References}

[1] J Kim H., Liang Y., Sakong K.M., Choi J.H. and CheolBak Y.: Temperature effect on the pressure drop across the cake of coal gasification as formed an a ceramic filterPowder Technology 181, 2008, 67-73

[2] Bergman W., Taylor R.D., Miller H.H., BIermann A.H., Hebard H.D. , DaRoaza R.A. and Lum B.Y.: Enhanced filtration program at LLL-a progress report- in: $15^{\text {th }}$ DOE NACC1978, pp.1058-1099

[3] Vendel J., Mulcey P.H. and Letourneau P.: Aerosol penetration inside HEPA filtration media- in: $21^{\text {st }}$ DOE/NRC NaCC-1990, pp, 799-808

[4] Letourneau P., Renaudin V. and Vendel J.: Effect of the particle penetration inside the filter medium on the HEPA filter pressure drop, in: $22^{\text {nd }}$ DOE/NRC NACC, 1992, pp, 128142

[5] Novick V.J., Klassen J.F., Monsone P.R. and Long T.A.: Predicting mass loading as a function of pressure difference across profiler/HEAP filter systems- in: $22^{\text {nd }}$ DOE/NRC NACC-1992, pp,554-573

[6] Thomas D., Contal P. ,Renaudin V., Penicot P., Leclerec D. and Vendel J.: Modeling pressure drop in heat filters during dynamic filtration- PII:S0021-8502(98)0036-6

[7] Cai Q.: A study of air filters by computational fluid dynamics, MSc thesis, School of Mechanical Aerospace Engineering-Oklahoma State University-USA-1993.

[8] Tebbutt C.B.: Computational fluid dynamic model of flow through air filter plates, MSc Thesis Mechanical and Aerospace engineering-Oklahoma State University-USA-1995

[9] Chen D.R., Pui D.Y.H. and Liu B.Y.H.: Optimization of pleated filters designs using a finite-element numerical model- Aerosol Science and Technology, 24- 1995, p.579

[10] Fabbro L.D., Laborde J.C., Merlin P. and Ricciardi L.: Air flows and pressure drop modeling for different pleated industrial filters- Research article, p.35-40

[11] Awni Y. and Otoom A.: Prediction of the collection efficiency, the porosity, and the pressure drop across filter cake in particulate air filtration- atmospheric Environment 392005, 51-57

[12] Gimbun J., Chuah T.G., Frakhru 'I-Razi A. and Choong T.: The influence of the temperature and inlet velocity on cyclone pressure drop: a CFD study-Chemical Engineering and Processing 44- 2005, 7-12

[13] Raynal L., Boyer C. and Ballaguetn J.P.: Liquid holdup and pressure drop determination in structured packing with CFD simulations-Can. J. Chem. 82- 2004, 871879

[14] Yang Z. and Zhu J.: A new k- $\varepsilon$ eddy viscosity model for high Reynolds number turbulent flows, Comp- Fluids 24- 1995, 227-238 